\title{
L- $\infty$ Norm Optimization for FIR Filter Design with Linear Phase in Passband by SDP Method
}

\author{
Haijiang $\mathrm{Hu}^{\mathrm{a}}$, Shaojing Song, Yu Chai and Yumei Gong \\ College of Engineering, Shanghai Polytechnic University, Shanghai 201209, China \\ ahjhu@sspu.edu.cn
}

\begin{abstract}
An improved optimization method for the FIR filter design is proposed., which uses SDP method to solve the filter design. The aim of optimization is least group delay in passband while the constraint conditions include the magnitude errors in all frequency domain. The experiment results show this method has good performance on the magnitude error and the linear phase in the passband. Therefore, this method can be used in the field of digital signal processing.
\end{abstract}

Keywords: FIR; convex optimization; SDP; linear phase.

\section{Introduction}

FIR filter is an important type of filter in the field of signal processing because of its inherent stability, simpler structure, and easier implementation on linear phase [1]. The linear phase is an important property of filters in many applications. If the coefficients of a FIR filter are odd symmetry or even symmetry, this FIR filter has the linear phase. The linear phase in entire frequency domain can be easily achieved using the classic design method for FIR filters. In the process of filter design, the convex programming (CP) have already been a hot research for recent years, such as semi-definite programming (SDP) [2]. SDP is a branch of CP methods, and is used for FIR filters design with presetting amplitude and phase responses. The advantages of convex optimization algorithms are to solve the optimization problem with a mix of linear and convex quadratic constraint conditions. Therefore we can consider more constraint conditions for filter design by CP. However, the symmetric coefficients are not realized by all FIR design methods, especially in the methods based on the optimizing theories. Some optimization methods can obtain very good amplification-frequency characteristic, but not realize the linear phase. Therefore how to design an asymmetric coefficient FIR filter with excellent amplification-frequency characteristic and linear phase is an interesting study[3]-[6]. In this paper we focus on FIR design with the asymmetric coefficients and the linear phase in the passband. SDP method is applied in phase optimization in the process of our FIR design.

The paper is organized as follows. In Section 2 we introduce the constraint conditions and the optimizing objective. We explain the realization method in Section 3 and introduce experiment and performance analysis in Section 4. Finally we summarize our results in Section 5.

\section{Constraint Conditions in FIR Design}

For a given pre-setting FIR filter with frequency response $H_{d}(\omega)$, we want to design a FIR filter with $N$ order that is shown as follows:

$$
H_{d}\left(c, e^{j \omega}\right)=\sum_{n=0}^{N} h_{n} e^{-j n \omega},
$$

where $c=\left[h_{0} h_{1} h_{2} \cdots h_{N}\right]^{T}$ and $\mathrm{c}$ is the initial coefficient. Therefore the group delay is defined as follows: 


$$
\begin{aligned}
\tau_{h}\left(c, e^{j \omega}\right) & =-\frac{d}{d \omega}\left[\arg \left(H_{d}\right)\right] \\
& =-\frac{d}{d \omega}\left[\frac{c^{T} B}{c^{T} A}\right],
\end{aligned}
$$

where

$$
\begin{gathered}
A=[1 \cos \omega \cdots \cos N \omega]^{T} \text { and } \\
B=[0-\sin \omega-\sin 2 \omega \cdots-\sin N \omega]^{T} .
\end{gathered}
$$

Then we can obtain

$$
\tau_{h}\left(c, e^{j \omega}\right)=\frac{\left(c^{T} A^{\prime}\right)\left(c^{T} B\right)-\left(c^{T} B^{\prime}\right)\left(c^{T} A\right)}{\left(c^{T} A\right)^{2}} .
$$

The group delay deviation is defined as follows:

$$
e_{g}\left(x, e^{j \omega}\right)=\tau_{h}\left(c, e^{j \omega}\right)-\tau,
$$

where $\tau$ is group delay variable which can be optimized, and $x=\left[C^{T} \tau\right]^{T}$.

If $x_{k}$ is the value of $x$ after $k$ th iteration by using the optimization methods, we can get the group delay deviation $e_{g}\left(x_{k}, e^{j \omega}\right)$ which is stated as follows:

$$
e_{g}\left(x_{k}+\delta, e^{j \omega}\right) \approx e_{g}\left(x_{k}, e^{j \omega}\right)+\nabla e_{g}\left(x_{k}, e^{j \omega}\right)^{T} \delta
$$

Therefore when $\|\delta\|$ is smaller, the group delay deviation is more accurate obviously. If $\omega_{p l}$ and $\omega_{p h}$ are lower and upper edges of the passband, the passband group-delay deviation for the kth iteration is shown as follows:

$$
\begin{aligned}
E^{(g d)}(k) & =\int_{\omega_{p l}}^{\omega_{p h}} \mid e_{g}\left(x_{k+1}, e^{j \omega}\right) d \omega \\
& \approx \kappa_{g} \sum_{i=1}^{N} \mid e_{g}\left(x_{k+1}, e^{j \omega_{i}}\right), \omega_{i} \in \Psi_{p} \\
& \approx \sum_{i=1}^{N}\left|\kappa_{g} e_{g}\left(x_{k}, e^{j \omega_{i}}\right)+\kappa_{g} \nabla e_{g}\left(x_{k}, e^{j \omega_{i}}\right)^{T} \delta\right|
\end{aligned}
$$

where $\Psi_{p} \in\left\lfloor\omega_{p l}, \omega_{p h}\right\rfloor$ is the frequency sampling point sets in the passband, $\kappa_{g}$ is a constant, respectively. Therefore, if $\mathrm{L}-\infty$ norm is used, we can get

$$
E^{(g d)}(k) \approx\left\|C_{k} \delta+D_{k}\right\|_{\infty}
$$


where $C_{k}=\left[\begin{array}{c}\kappa_{g} \nabla e_{g}\left(x_{k}, e^{j \omega_{1}}\right)^{T} \\ \vdots \\ \kappa_{g} \nabla e_{g}\left(x_{k}, e^{j \omega_{p}}\right)^{T}\end{array}\right], D_{k}=\left[d_{1} d_{2} \cdots d_{N p}\right]^{T}$, and $d_{i}=\kappa_{g} e_{g}\left(x_{k}, e^{j \omega_{i}}\right)$, respectively.

Lower amplitude error in passband is an important target in the filter design. The passband error function is defined as

$$
e_{h}\left(c_{k}, e^{j \omega}\right)=\left|H\left(c_{k}, e^{j \omega}\right)\right|^{2}-\left|H_{d}(\omega)\right|^{2}, \omega \in \Psi_{p}
$$

We assume the ideal amplitude response is uniformization in the passband. Then Eq.8 is described as

$$
e_{h}\left(c_{k}, e^{j \omega}\right)=\left|H\left(c_{k}, e^{j \omega}\right)\right|^{2}-1, \omega \in \Psi_{p}
$$

Using the same approach as Eqs.5, 6 and 7, we can get $E^{(p b)}(k)$ as follows:

$$
\begin{aligned}
E^{(p b)}(k) & \approx \kappa_{p b} \sum_{i=1}^{N}\left|e_{h}^{(p b)}\left(c_{k+1}, e^{j \omega_{i}}\right)\right|, \omega_{i} \in \Psi_{p} \\
& \approx \sum_{i=1}^{N}\left|\begin{array}{c}
\kappa_{p b} e_{h}^{(p b)}\left(c_{k}, e^{j \omega_{i}}\right)+ \\
\kappa_{p b} \nabla e_{h}^{(p b)}\left(c_{k}, e^{j \omega_{i}}\right)^{T} \delta \mid
\end{array}\right|
\end{aligned}
$$

where $\delta=\left[\delta_{c}^{T} \delta_{\tau}\right]^{T}, \delta_{c}$ is the vector update for $c_{k}, \delta_{\tau}$ is the scalar update for $\tau$, and $\kappa_{p b}$ is a constant, respectively. Therefore, if L- $\infty$ norm is used, $E^{(p b)}(k)$ is stated as follows:

$$
E^{(p b)}(k) \approx\left\|D_{k}^{(p b)} \delta+f_{k}^{(p b)}\right\|_{\infty}
$$

Where

$$
\begin{aligned}
D_{k}^{(p b)} & =\left[\begin{array}{cc}
\kappa_{p b} \nabla e_{h}^{(p b)}\left(c_{k}, e^{j \omega_{1}}\right)^{T} & 0 \\
\vdots & \vdots \\
\kappa_{p b} \nabla e_{h}^{(p b)}\left(c_{k}, e^{j \omega_{N_{p}}}\right)^{T} & 0
\end{array}\right], \omega_{i} \in \Psi_{p} \\
f_{k}^{(p b)} & =\left[f_{1}^{(p b)} f_{2}^{(p b)} \cdots f_{N_{p}}^{(p b)}\right] \\
f_{i}^{(p b)} & =\kappa_{p b} e_{h}^{(p b)}\left(c_{k}, e^{j \omega_{i}}\right) .
\end{aligned}
$$

The amplitude responsess in the stopband and transition band are also considered as follows:

$$
e_{h}\left(c_{k}, e^{j \omega}\right)=\left|H\left(c_{k}, e^{j \omega}\right)\right|^{2}-\left|H_{d}(\omega)\right|^{2}, \omega \in \Psi_{s}
$$

and

$$
e_{h}\left(c_{k}, e^{j \omega}\right)=\left|H\left(c_{k}, e^{j \omega}\right)\right|^{2}-\left|H_{d}(\omega)\right|^{2}, \omega \in \Psi_{t}
$$

In the ideal filter, the amplitude responses in the stopband and transition band are zero. Therefore 


$$
e_{h}\left(c_{k}, e^{j \omega}\right)=\left|H\left(c_{k}, e^{j \omega}\right)\right|^{2}, \omega \in \Psi_{s},
$$

and

$$
e_{h}\left(c_{k}, e^{j \omega}\right)=\left|H\left(c_{k}, e^{j \omega}\right)\right|^{2}, \omega \in \Psi_{t}
$$

The same to analysis on the passband, we can get

and

$$
E^{(s b)}(k) \approx\left\|D_{k}^{(s b)} \delta+f_{k}^{(s b)}\right\|_{\infty},
$$

$$
E^{(t b)}(k) \approx\left\|D_{k}^{(t b)} \delta+f_{k}^{(t b)}\right\|_{\infty}
$$

Where

$$
\begin{aligned}
& D_{k}^{(s b)}=\left[\begin{array}{cc}
\kappa_{s b} \nabla e_{h}^{(s b)}\left(c_{k}, e^{j \omega_{1}}\right)^{T} & 0 \\
\vdots & \vdots \\
\kappa_{s b} \nabla e_{h}^{(s b)}\left(c_{k}, e^{j \omega_{N_{s}}}\right)^{T} & 0
\end{array}\right], \omega_{i} \in \Psi_{s} \\
& f_{k}^{(s b)}=\left[f_{1}^{(s b)} f_{2}^{(s b)} \cdots f_{N_{s}}^{(s b)}\right] \text {, } \\
& f_{i}^{(s b)}=\kappa_{s b} e_{h}^{(s b)}\left(c_{k}, e^{j \omega_{i}}\right), \\
& D_{k}^{(t b)}=\left[\begin{array}{cc}
\kappa_{t b} \nabla e_{h}^{(t b)}\left(c_{k}, e^{j \omega_{1}}\right)^{T} & 0 \\
\vdots & \vdots \\
\kappa_{t b} \nabla e_{h}^{(t b)}\left(c_{k}, e^{j \omega_{N_{t}}}\right)^{T} & 0
\end{array}\right], \omega_{i} \in \Psi_{t} \\
& f_{k}^{(t b)}=\left[\begin{array}{lll}
f_{1}^{(t b)} & f_{2}^{(t b)} \cdots f_{N_{t}}^{(t b)}
\end{array}\right] \text {, } \\
& f_{i}^{(t b)}=\kappa_{t b} e_{h}^{(t b)}\left(c_{k}, e^{j \omega_{i}}\right) \text {. }
\end{aligned}
$$

Both $\kappa_{s b}$ and $\kappa_{t b}$ are constants.

Based on the above analysis, an optimization problem can be created as follows: Minimize

$$
\left\|C_{k} \delta+D_{k}\right\|_{\infty}
$$

Subject to

$$
\begin{gathered}
\left\|D_{k}^{(p b)} \delta+f_{k}^{(p b)}\right\|_{\infty}<\Gamma_{p b}, \\
\left\|D_{k}^{(s b)} \delta+f_{k}^{(s b)}\right\|_{\infty}<\Gamma_{s b}, \\
\left\|D_{k}^{(t b)} \delta+f_{k}^{(t b)}\right\|_{\infty}<\Gamma_{t b}, \\
\|\delta\|_{\infty}<\Gamma_{\min } .
\end{gathered}
$$


In this optimization problem, $\Gamma_{p b}, \Gamma_{s b}, \Gamma_{t b}$, and $\Gamma_{\min }$ are pre-setting constants. Therefore, we can use this optimization problem to describe the FIR filter design with the minimum group delay in passband.

\section{Implementation}

Obviously, the above optimization problem can be solved by SDP method. Therefore we can describe the implementation process as follows:

Step 1:Design a FIR filter with the presetting amplitude in the passband and the stopband by using some optimization methods.

Step 2: Define $\Gamma_{p b}, \Gamma_{s b}, \Gamma_{t b}$, and $\Gamma_{\min }$.

Step 3: According to the presetting group delay in passband, construct $C_{k}$ and $D_{k}$.

Step 4: According to the constraint conditions, construct $D_{k}^{(p b)}, D_{k}^{(s b)}, D_{k}^{(t b)}, f_{k}^{(p b)}, f_{k}^{(s b)}$, and $f_{k}^{(t b)}$.

Step 5: Use SDP method to solve this optimize problem, and obtain the result $\delta_{\min }$.

Step 6: According to the result $\delta_{\min }$, refresh the filter coefficient $c$.

\section{Experiments}

In order to test the proposed method, we design a FIR filter to testify the performance of this method. Our all filter designs are realized by using SeDuMi toolbox 1.3 in Matlab R2009b. In our design, we use SDP to calculate the coefficients based on the least-squares. In the process of optimization, we choose 100 sample points in the frequency domain and define $\delta$ is 0.01 . Moreover, $\kappa_{g}, \kappa_{p b}, \kappa_{t b}$, and $\kappa_{s b}$ are $1, \Gamma_{p b}, \Gamma_{s b}$, and $\Gamma_{t b}$ are $0.1, \Gamma_{\min }$ is 0.001 , respectively.

In this example, we design a low-pass filter with 20 orders which is described as follows:

$$
H(j \omega)=\left\{\begin{array}{cc}
e^{-j 9.2 \omega}, \omega \in[0,0.3 \pi] \\
0, \quad \omega \in[0.6 \pi, \pi]
\end{array} .\right.
$$

The ideal group delay in this filter is 9.2. The passband is $[0,0.3 \pi]$ while the stopband is $[0.6 \pi, \pi]$. In our design, we choose 100 sample points in the frequency domain. Fig.1 shows the performance of design result by our proposed method. From Fig.1, we can know the magnitude-frequency characteristic meets the requirement while the group delay in passband is the approximate linearization. Table. 1 shows the passband error $\delta_{p}$, the stopband error $\delta_{s}$, and the group delay error $\delta_{g}$ from our proposed method for the comparison with [5]. From this Table, we can see the performance on the passband error, the stopband error, and the group delay error in our proposed method are better than the performance on passband error and group delay error in [5]. 

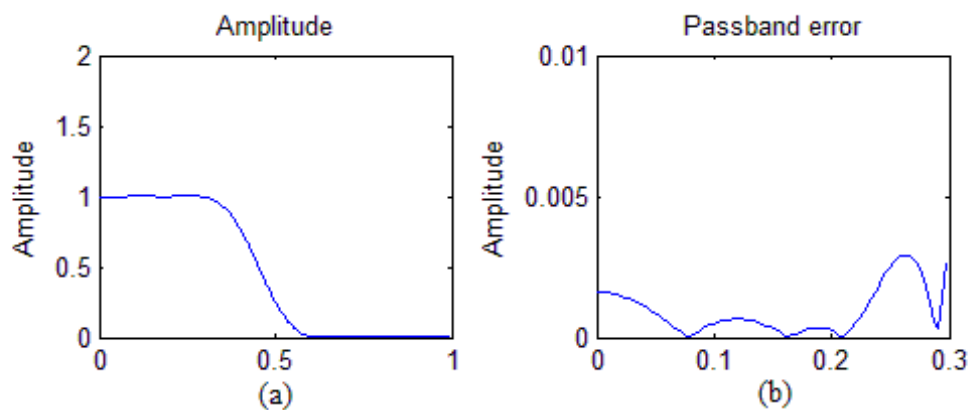

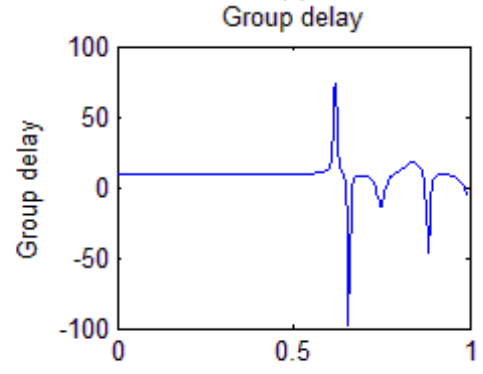

(c)

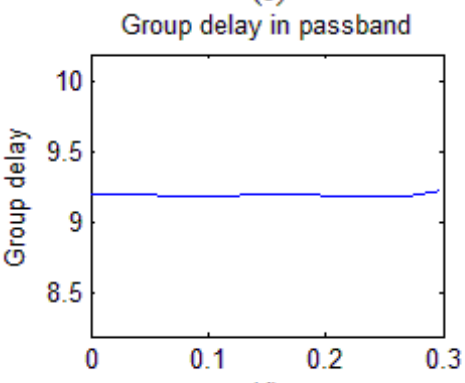

(d)

Fig.1 Performance of design result for Example 1: (a) Amplitude; (b) Passband error; (c) Group delay; (d) Group delay in passband.

Table 1. Performance comparison in example 1

\begin{tabular}{cccc}
\hline \multirow{2}{*}{ Design method } & \multicolumn{3}{c}{ Performance } \\
\cline { 2 - 4 } & $\delta_{p}(\mathrm{~dB})$ & $\delta_{s}(\mathrm{~dB})$ & $\delta_{g}$ \\
\hline Proposed method & 0.0065 & 59.4537 & 0.0124 \\
\hline Design 1 in [5] & 0.0160 & 52.3450 & 0.0593 \\
\hline Design 2 in [5] & 0.0190 & 52.7930 & 0.0518 \\
\hline
\end{tabular}

\section{Conclusion}

In this paper we introduce an improved method to solve FIR filter design with low magnitude error and linear phase in passband. This method uses SDP method to optimize the filter coefficient based on least group delay in passband. Moreover, in the constraint conditions, the low magnitude errors in all frequency domains are used for the optimization. The experiments testify this method has good performance on the passband error and group delay error in the passband. Therefore this method is an efficient algorithm for FIR filter optimization which can be used in the digital signal processing widely.

\section{Acknowledgements}

The generous support of the Discipline Construction Fund of SSPU (XXKZD1605) and the Construction of University Enterprise Cooperation Automobile Electronic Joint Experiment Center (A11NH182016) are gratefully acknowledged.

\section{References}

[1]. G. Beylkin, R. D. Lewis, and L. Monzón, "On the design of highly accurate and efficient IIR and FIR filters”, IEEE Trans. Signal Process., vol. 60, no. 8, pp. 4045-4054, Aug. 2012.

[2]. R. C. Longpiur, D. J. Shpak, and A. Antoniou, "Improved design method for nearly linear-phase IIR filters using constrained optimization," IEEE Trans. Signal Process., vol. 61, no. 4, pp. 895906, Feb. 2013. 
[3]. C. Wu, D. Gao, and K. L. Teo, "A direct optimization method for low group delay FIR filter design”, Signal Process., vol. 93, pp.1764-1772 Jan. 2013.

[4]. X. Lai, C. Lai, and R. Zhao, "An iterative approach to near-uniform group-delay error design of FIR filters”, IEEE Signal Process. Lett., Vol. 18, No. 2, pp.107-110, Feb. 2011.

[5]. K. M. Chui, S. C. Chan, and K. S. Yeung, "Design of FIR digital filters with prescribed flatness and peak error constraints using second-order cone programming," IEEE Trans. Circuits Syst. II, vol. 52, no. 9, pp.601-605, Sep. 2005.

[6]. X. Lai, "Optimal design of nonlinear-phase FIR filters with prescribed phase error," IEEE Trans. Signal Process., vol. 57, no. 9, pp.3399-3410, Sep. 2009. 